\title{
Reseda luteola L. en el estado de Aguascalientes, México
}

\author{
Reseda luteola L. in the state of Aguascalientes, Mexico
}

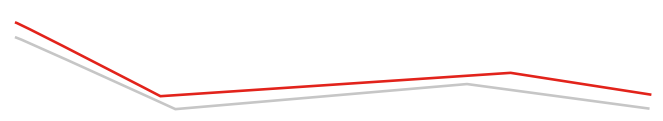

Manuel Higinio Sandoval-Ortega*®, María Elena Siqueiros-Delgado*

Sandoval-Ortega, M. H., \& Siqueiros-Delgado, M. E. (2019). Reseda luteola L. en el estado de Aguascalientes, México. Investigación y Ciencia de la Universidad Autónoma de Aguascalientes, 27(76), 5-10.

RESUMEN

El estado de Aguascalientes se encuentra ubicado en la zona centro norte del país y ocupa alrededor del $0.3 \%$ de la superficie nacional. Florísticamente pertenece a la región Xerofítica Mexicana y, en menor proporción, a la Mesoamericana de Montaña. El objetivo de este trabajo es realizar el tratamiento taxonómico de la familia Resedaceae en el estado de Aguascalientes, donde está representada por Reseda luteola L., una especie introducida originaria del viejo mundo donde se utiliza para la producción de colorantes y que es una maleza distribuida en varios estados de la República Mexicana. En Aguascalientes es una planta común, principalmente como elemento de vegetación secundaria derivada de matorral xerófilo, que prospera en orillas de camino y en áreas verdes urbanas.

\section{ABSTRACT}

The state of Aguascalientes is located in the north central zone of the country and occupies around

Palabras clave: flora; taxonomía; biodiversidad; botánica; vegetación; ecosistemas.

Keywords: flora, taxonomy, biodiversity, botany, vegetation, ecosystems.

Recibido: 2 de junio de 2018, aceptado: 18 de septiembre de 2018

* Herbario HUAA, Departamento de Biología, Centro de Ciencias Básicas, Universidad Autónoma de Aguascalientes. Avenida Universidad 940, Ciudad Universitaria, C. P. 20131, Aguascalientes, Aguascalientes, México. Correo electrónico: m.higinio.s@hotmail.com; masiquei@correo. vaa.mx. ORCID: http://orcid.org/0000-0003-1396-9024; http://orcid. org/0000-0002-0865-3589.

$\triangle \quad$ Autor para correspondencia
$0.3 \%$ of the national surface. About floral territory, Aguascalientes belongs to the Mexican Xerophytic region and, to a lesser extent, to the Mesoamerican of Mountain. The objective of this work is to perform the taxonomic treatment of the Resedaceae family in the state of Aguascalientes, where it is represented by Reseda luteola L., a species which was introduced from the old world where it is used for the production of dyes and which is a weed distributed in several states of the Mexican Republic. In Aguascalientes, it is a common plant, mainly as an element of secondary vegetation derived from xerophilous scrub, which thrives on the banks of the road and in urban green areas.

\section{INTRODUCCIÓN}

El estado de Aguascalientes se encuentra ubicado en la zona centro norte del país, posee una extensión de $5,616 \mathrm{~km}^{2}$, que representan aproximadamente $0.3 \%$ de la superficie nacional; consta de once municipios: Aguascalientes, Asientos, Calvillo, Cosío, El Llano, Jesús María, Pabellón de Arteaga, Rincón de Romos, San Francisco de los Romo, San José de Gracia y Tepezalá (INEGI, 2016).

Florísticamente, pertenece a dos regiones: la Xerofítica Mexicana y, en menor proporción, la Mesoamericana de Montaña (Rzedowski, 2006). La vegetación templada es la más ampliamente distribuida en el estado, ocupa la zona montañosa o picos aislados y cubre $30.48 \%$ de la superficie total de Aguascalientes; alrededor de $30 \%$ es vegetación primaria; se distinguen diferentes asociaciones de bosques de encino, pino y mixtos, además de matorrales y pastizales templados. La vegetación 
issn 1665-4412, e-issn 2521-9758

Sandoval-Ortega, M. H., \& Siqueiros-Delgado, M. E. subtropical ocupa solo $6.58 \%$ de la superficie total del estado, donde $16.8 \%$ se encuentra en condición primaria, aunque con alto grado de disturbio, se distribuye en el suroeste de Aguascalientes y está representada por el bosque tropical bajo caducifolio y el matorral subtropical en las partes más accesibles, el cual es considerado como vegetación secundaria (Siqueiros-Delgado, Rodríguez-Ávalos, MartínezRamírez, \& Sierra-Muñoz, 2016).

La vegetación xerófila ocupa $21.52 \%$ de la superficie total de Aguascalientes; alrededor de 55\% tiene vegetación primaria, especialmente pastizal natural y se distribuye principalmente en las planicies centrales formada por comunidades de mezquitales, matorrales y pastizales con diferentes asociaciones vegetales. Por último, la vegetación hidrófila ocupa una reducida superficie, con $0.37 \%$ del área y $35 \%$ se conserva con vegetación primaria (SiqueirosDelgado, Rodríguez-Ávalos, Martínez-Ramírez, \& Sierra-Muñoz, 2016).

Aunque existen estudios sobre flora de Aguascalientes, algunas familias necesitan ser revisadas para determinar el número de especies y la situación de sus poblaciones. Entre estas familias se encuentra Resedaceae, descrita por primera vez por Martinov en 1820 y está integrada por ocho géneros y 96 especies (Stevens, 2001), que se distribuyen en las regiones templadas del hemisferio norte, principalmente en Europa, Asia y África. Las especies de esta familia prefieren lugares soleados como estepas, sabanas, desiertos y varios de sus integrantes pueden ser vistos como invasores de zonas de reciente disturbio o como malezas ruderales y arvenses (Abdallah, 1967).

A partir de agosto de 2012 hasta abril de 2017 se llevó a cabo el proyecto JF140 de CONABIO "Inventario florístico de familias selectas de dicotiledóneas del estado de Aguascalientes" y actualmente se está trabajando en la flora dicotiledónea del estado, de donde se desprende el presente trabajo, con el objetivo de realizar el tratamiento taxonómico de la familia Resedaceae para el estado.

\section{MATERIALES Y MÉTODOS}

Se llevaron a cabo recolectas en 195 puntos distribuidos en todo el estado siguiendo la metodología propuesta por Engelmann (1986). En cada uno de estos sitios se tomaron coordenadas geográficas con base en Datum WGS 84, se registró el tipo de vegetación de acuerdo con Rzedowski (2006) y con SiqueirosDelgado, Rodríguez-Ávalos, Martínez-Ramírez, Sierra-Muñoz y García-Regalado (2017). El material fue identificado por medio de claves taxonómicas especializadas (Abdallah, 1967; Calderón de Rzedowski, 1995, 2001; Moreno \& Escamilla, 1984; Valdés-Bermejo, 1993) y cotejado con ejemplares depositados en el Herbario de la Universidad Autónoma de Aguascalientes (HUAA). Con base en los ejemplares y bibliografía revisados se elaboraron descripciones taxonómicas de familia, género y especie, así como un mapa de distribución por medio del programa QGIS.

\section{RESULTADOS}

\section{Resedaceae Martinov}

Plantas herbáceas, subarbustivas, arbustivas o arborescentes, glabras o puberulentas. Tallos erectos a ascendentes, simples o ramificados. Hojas caulinares o en una roseta basal, pecioladas, sésiles o subsésiles, alternas o fasciculadas; láminas simples, enteras o pinatífidas, estípulas presentes y modificadas en glándulas, venación pinnada. Inflorescencias terminales rara vez axilares, espigas o racimos, brácteas presentes. Flores sésiles o pediceladas, ligeramente zigomorfas, hermafroditas 0 unisexuales, pequeñas. Cáliz con 2-8 sépalos libres o brevemente connados en la base, iguales o subiguales, persistentes o deciduos en fruto. Corola presente o ausente, cuando presente de 2-8 pétalos pequeños y poco evidentes, libres o connados, lobulados a laciniados; estambres $3-40$, con frecuencia colocados a un lado de un disco hipógino, filamentos libres o connados basalmente, persistentes o deciduos, anteras bitecas, de dehiscencia longitudinal; nectario presente o ausente, cuando presente en forma de disco, asimétrico; ovario de 2-8 carpelos libres o connados, unilocular, con 2-8 dientes apicales con el mismo número de estigmas sésiles; óvulos por lo común numerosos, placentación parietal, campilotropos. Fruto generalmente una cápsula angular, membranosa o papirácea, ovada, oblongo-ovada, cilíndrica o subglobosa, de dehiscencia apical, rara vez una baya. Semillas reniformes, testa lisa $u$ ornamentada.

Familia de ocho géneros y 96 especies distribuidas principalmente en el hemisferio norte y unas pocas en el sur de África (Stevens, 2001). Para México se reportan tres géneros (considerando Forchhammeria) y un total de nueve especies (Villaseñor, 2016). Sólo un género reportado para Aguascalientes. 


\section{Reseda L.}

Plantas herbáceas anuales o perennes, en ocasiones subarbustivas, glabras, pilosas o papilosas. Tallos simples o ramificados distalmente, erectos o ascendentes, lisos o estriados longitudinalmente. Hojas en roseta basal y caulinares, las de la roseta basal pecioladas, las caulinares alternas, subsésiles o sésiles, láminas simples o pinnatífidas, estípulas glandulares cónicas, espiniformes, base truncada, atenuada o decurrente, ápice agudo, redondeado, obtuso o mucronado, margen entero, ondulado - dentado. Inflorescencias terminales, racimos o espigas, con una bráctea en la base de cada flor. Flores pediceladas, hermafroditas; cáliz de 4-6(8) sépalos, brevemente connados en la base, subiguales, persistentes o deciduos; corola presente pétalos 4-6(8), blancos, amarillos o anaranjados, desiguales, uno de ellos más grande que los otros; estambres 7-40, saliendo a un lado de un disco unilateral; disco nectarífero presente; ovario de (2) 3-4 (5) carpelos, con el mismo número de dientes apicales. Fruto capsular, anguloso, membranoso a papiráceo, ovado, oblongo-ovado, cilíndrico o subgloboso, abriéndose antes de la madurez de las semillas. Semillas de testa papilosa, rugosa o lisa.

Género integrado por 68 especies distribuidas principalmente en el Mediterráneo y norte de África, ampliamente naturalizado en zonas templadas y subtropicales del mundo. Sólo una especie reportada para México y, en consecuencia, para Aguascalientes.

Reseda luteola L., Species Plantarum 1: 448 (1753) (figuras 1 y 2 )

Planta herbácea, anual, glabra. Tallos erectos, simples o ramificados de $40-80$ (120) cm de alto, estriados longitudinalmente. Hojas de la roseta basal con peciolo de (0.5) $1-3(6) \mathrm{cm}$ de largo por $0.4-0.6 \mathrm{~cm}$ de ancho, estípulas 2-4 en la base de la lámina, espiniformes, de (1)2-3 mm de largo, láminas oblanceoladas a espatuladas, de 8-17(22) $\mathrm{cm}$ de largo y $2-3 \mathrm{~cm}$ de ancho, base atenuada a brevemente decurrente, ápice obtuso 0 redondeado, margen ondulado; hojas caulinares sésiles, más pequeñas que las basales, con 2 estípulas basales espiniformes de hasta $1 \mathrm{~mm}$ de largo, láminas lineares o lanceoladas de $2-5(7) \mathrm{cm}$ de largo por 0.5-0.7 cm de ancho, base truncada, ápice agudo, redondeado o mucronulado, margen por lo común entero, en ocasiones levemente ondulado. Inflorescencia en racimos, de $20-35 \mathrm{~cm}$

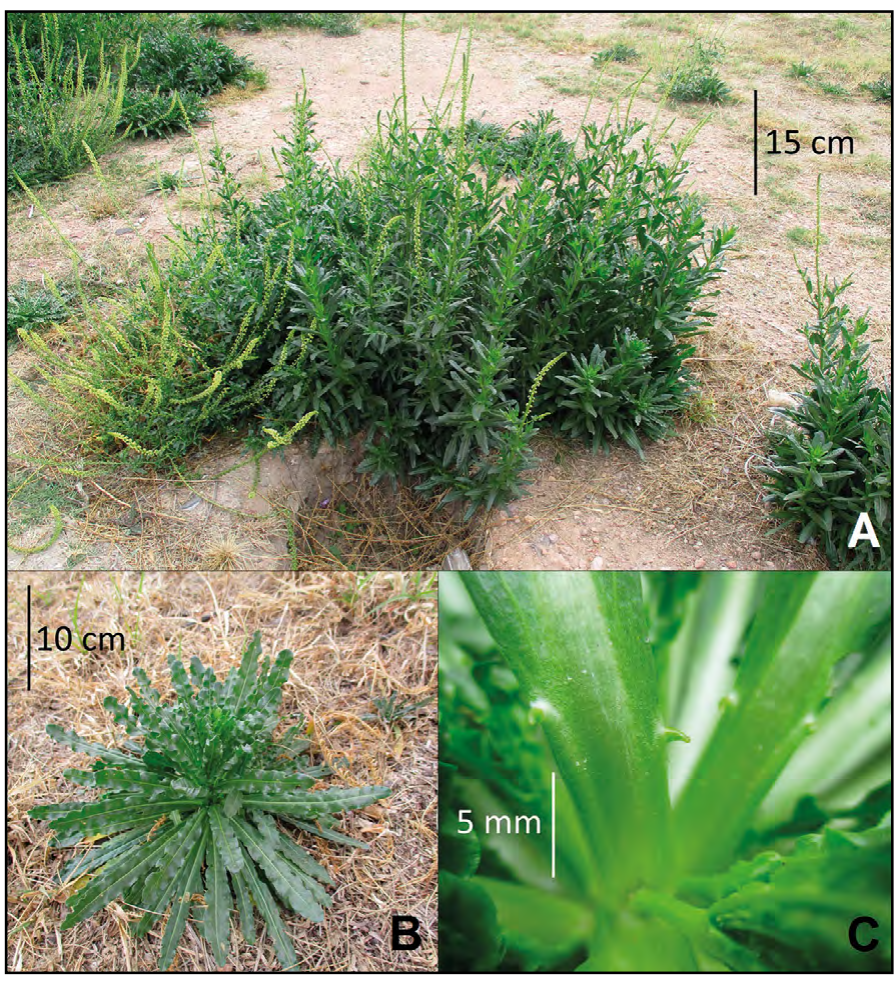

Figura 1. A) Vista general. B) Planta joven con roseta basal.

C) Detalle de estípulas.

Fotografías tomadas por los autores.

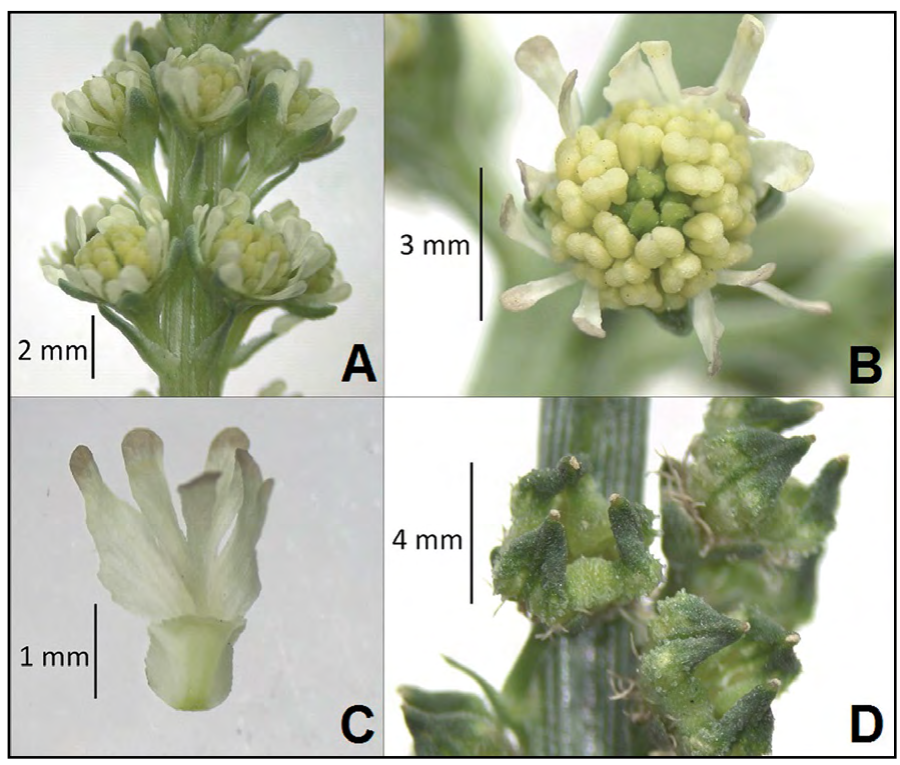

Figura 2. A) Detalle de inflorescencia. B) Detalle de flor. C) Vista adaxial de pétalo. D) Detalle de fruto.

Fotografías tomadas por los autores.

de largo con numerosas flores; brácteas subuladas (2)3-3.5 mm de largo, base truncada y ápice agudo, margen escarioso, persistentes en fruto. Flores 
issn 1665-4412, e-issn 2521-9758

Sandoval-Ortega, M. H., \& Siqueiros-Delgado, M. E.
8

cortamente pediceladas, pedicelos de 2-3(4) mm de largo; cáliz de 4 sépalos ovados a lanceolados, de 2-2.3 $\mathrm{mm}$ de largo por 0.8-1.2 $\mathrm{mm}$ de ancho; corola de 4 pétalos laciniados, de (2)3-4(5) $\mathrm{mm}$ de largo, con una membrana horizontal cercana a la base en la cara adaxial, blancos a amarillentos; estambres 20-30, filamentos de 1-1.5 mm de largo, blancos, persistentes en fruto, anteras de hasta $1 \mathrm{~mm}$ de largo; ovario tricarpelar, obovado, de $2.5-3 \mathrm{~mm}$ de largo, c. de $1.5 \mathrm{~mm}$ de ancho, superficie cubierta por papilas hialinas persistentes en fruto, 3 dientes apicales. Fruto capsular, subgloboso, de 4-5(6) mm de diámetro. Semillas reniformes, de $1 \mathrm{~mm}$ de largo marrón oscuras a negras, testa lisa y lustrosa.

Se le conoce localmente como "gualda" o "hierba del mosco". Su época de floración es desde finales de otoño hasta invierno, y se le puede encontrar en fruto a finales de enero y principios de febrero. Es de uso forrajero y, debido a que llega a cubrir grandes cantidades de terreno, constituye en muchos casos el único alimento para el ganado durante la temporada invernal. Se le llama hierba del mosco ya que en ésta época es muy visitada por las abejas (figura 3).

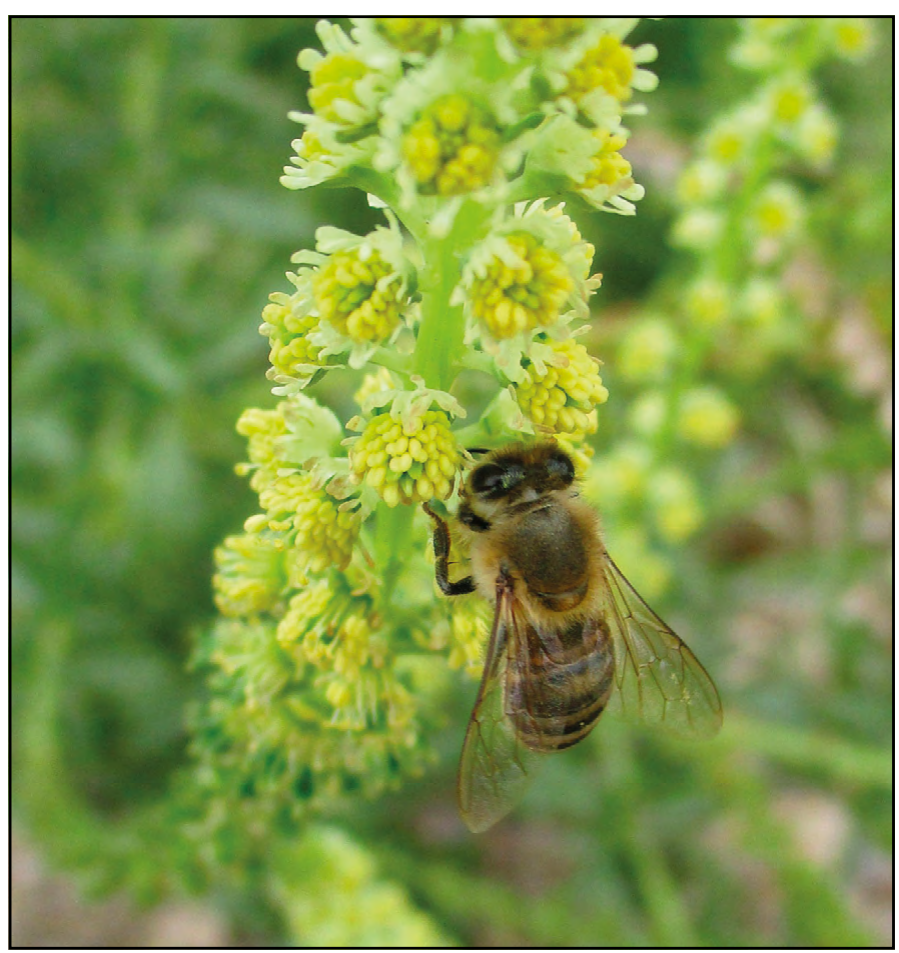

Figura 3. Reseda luteola L. siendo visitada por una abeja.

Fotografía tomada por los autores.
En Aguascalientes es una planta común, principalmente como elemento de vegetación secundaria derivada de matorral xerófilo, crece en orillas de camino y áreas verdes de zonas urbanas (figura 4).

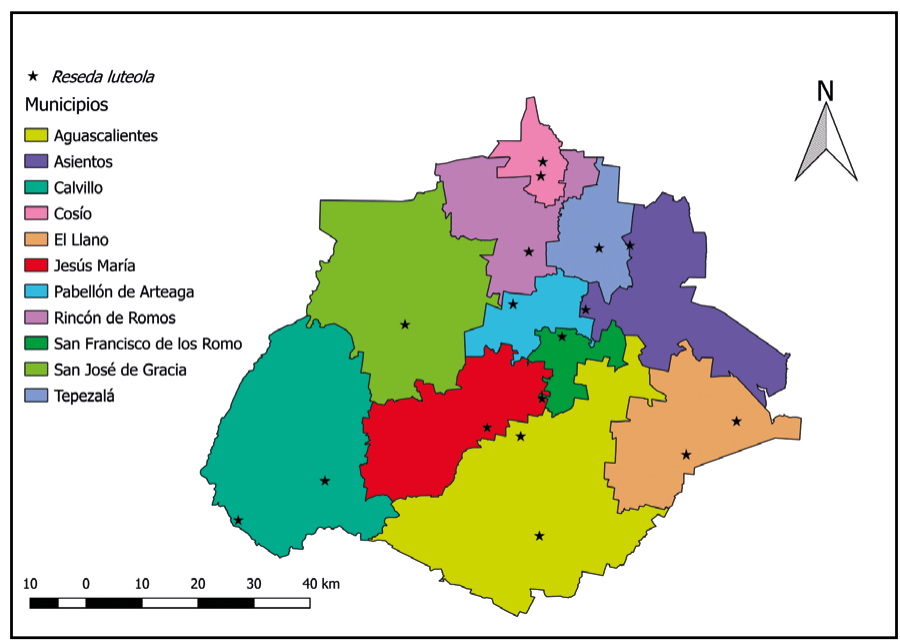

Figura 4. Distribución de Reseda luteola L. en el estado de Aguascalientes.

Elaboración propia.

Ejemplares examinados: Aguascalientes: Granja el Huizache, 500 metros al NE de San Antonio de Peñuelas, De la Cerda, 5323 (HUAA); Jardín Botánico Universidad Autónoma de Aguascalientes, De la Cerda, 4558 (HUAA). Asientos: Faldas del cerro del Chiquihuite, Siqueiros. M.E 2017 (HUAA); Cerro de Microondas, $22^{\circ} 13^{\prime} 5.8^{\prime \prime} \mathrm{N}$ 102 $2^{\prime}$ '50.3'W, SandovalOrtega 1010 (HUAA). Calvillo: $1.5 \mathrm{~km}$ al SE de Malpaso, Sandoval-Ortega 1013 (HUAA); Jaltiche de Abajo, Sandoval-Ortega 1014 (HUAA). Cosío: Pozo del Carrizal, SE de Cosío, De la Cerda, 5294 (HUAA). El Llano: El Tildio, $21^{\circ} 52^{\prime} 58.3^{\prime \prime} \mathrm{N} 102^{\circ} 2^{\prime} 3.1$ ' 'W, SandovalOrtega 1019 (HUAA); El Novillo, $1 \mathrm{~km}$ al NE de Palo Alto, 2156'12.1"N 10156'50"W, Sandoval-Ortega 1020 (HUAA). Jesús María: Viñedos San Marcos, Delgado J. s/n (HUAA); Curva de la doble A, orilla de la carretera, Esparza. S 13 (HUAA). Pabellón de Arteaga: El Milagro, ejido Santiago, De la Cerda, 5304 (HUAA). Rincón de Romos: Ejido de la Víbora $(1 \mathrm{~km}$ al $\mathrm{S}$ de Rincón de Romos), De la Cerda, 5285 (HUAA). San Francisco de los Romo: Rancho Villa de Guadalupe, Sandoval-Ortega 1018 (HUAA). San José de Gracia: Potrero de los López, 2205'30"N 102³1'16.4"W, Sandoval-Ortega 1015 (HUAA). Tepezalá: $1 \mathrm{~km}$ al S de Tepezalá, $22^{\circ} 12^{\prime} 58.0^{\prime \prime} \mathrm{N} 102^{\circ} 11^{\prime} 7.2^{\prime \prime} \mathrm{W}$, SandovalOrtega 1012 (HUAA). 
DISCUSIÓN

En México se han realizado trabajos taxonómicos para esta familia en la región del Bajío (Calderón de Rzedowski, 1995), el valle de México (Calderón de Rzedowski, 2001), Guerrero (Fonseca, 2005) y Veracruz (Nee, 1985), se han reportado hasta la fecha los géneros Forchhammeria Liebm., Oligomeris Cambess. y Reseda L. en el país.

Forchhammeria fue anteriormente considerado dentro de la familia Capparaceae (Newman, 2007; Standley, 1924) y actualmente se incluye en Resedaceae (APG IV, 2016; Stevens, 2001). Este género cuenta con alrededor de 10 especies (Newman, 2007), nueve de ellas distribuidas en México, de donde siete son endémicas (Villaseñor, 2016).

Por otro lado, Oligomeris está integrado por tres especies, dos de ellas restringidas al suroeste de África y solo O. linifolia posee una distribución amplia y disyunta, encontrándose desde el norte de África hasta el suroeste de Asia, y en el sureste de Estados Unidos y norte de México, donde también se le considera nativa (Martín-Bravo, Vargas, \& Luceño, 2009).

Por último, el género Reseda es el que incluye el mayor número de especies dentro de la familia Resedaceae a nivel global, con un total de 68 (Stevens, 2001). Para el continente americano se han reportado Reseda alba L., R. Iutea L., R. Iuteola L., y $R$. odorata L., todas estas introducidas (Martín-Bravo et al., 2009), para México se reporta solo R. Iuteola L. (Villaseñor \& Espinosa-García, 2004).

Reseda luteola L. es una especie proveniente de Europa, donde se le cultivaba por su colorante amarillo (Valdés-Bermejo, 1993) y posiblemente fue introducida en el país con el mismo fin.

De acuerdo con Angelini, Bertoli, Rolandelli y Pistelli (2003) Reseda luteola produce flavonoides en sus partes aéreas, principalmente en las hojas e inflorescencias, el más importante de estos es la luteolina, un pigmento amarillo utilizado para teñir lana y seda desde tiempos antiguos. En países como Turquía sigue siendo utilizada para para teñir alfombras y se le considera una especie de alto valor económico (Doğan, 2001).

Actualmente es una maleza distribuida en varios estados de la República Méxicana (Villaseñor \& Espinosa-García, 1998) y en Aguascalientes es común en tierras de cultivos como el ajo, alfalfa, avena, brócoli, cebolla y Lolium, en suelos generalmente franco arcillosos con un pH de 5.2 a 8.5 (De la CerdaLemus, 2002) y se le considera como una especie de importancia melífera, productora de néctar y polen (Franco-Olivares, Siqueiros-Delgado, \& HernándezAyala, 2012). Además de poseer valor para el ganado, principalmente caprino, ya que de acuerdo a Nagel et al. (2011), su alto contenido en proteína cruda y bajo contenido de fibra la convierten en una planta forrajera excepcional.

\section{CONCLUSIONES}

Reseda luteola L. es el único representante de la familia Resedaceae en el estado de Aguascalientes. Es una planta común que se distribuye principalmente en áreas con disturbio y como maleza arvense y ruderal en la zona árida y semiárida del estado, que es de importancia melífera, forrajera y que ha sido utilizada en el viejo mundo como colorante natural desde tiempos antiguos, sin embargo, en Aguascalientes no se le da este uso. 
- Abdallah, M. S. (1967). The Resedaceae. A taxonomical revision of the family. Mededelingen Landbouwho-geschool Wageningen, 67(8), 1-98.

- Angelini, L. G., Bertoli, A., Rolandelli, S., \& Pistelli, L. (2003). Agronomic potential of Reseda luteola L. as new crop for natural dyes in textiles production. Industrial Crops and Products, 17(3), 199-207. doi: 10.1016/S0926-6690(02)00099-7

- Angiosperm Phylogeny Group IV. (2016). An update of the Angiosperm Phylogeny Group classification for the orders and families of flowering plants: APG IV. Botanical Journal of the Linnean Society, 181 (1), 1-20. doi: 10.1111/boj.12385

- Calderón de Rzedowski, G. (1995). Resedaceae. Flora del Bajío y de Regiones Adyacentes, 35, 1-6.

(2001). Resedaceae. En G. Calderón \& J. Rzedowski (Eds.), Flora del Valle de México (2 ed., p. 213). México: Instituto de Ecología, A.C.-CONABIO.

- De la Cerda-Lemus, M. (2002). Malezas de Aguascalientes. México: Universidad Nacional Autónoma de México.

- Doğan, Y. (2001). A study on the autecology of Reseda lutea L. (Resedaceae) distributed in Western Anatolia. Turkish Journal of Botany, 25, 137-148.

- Engelmann, G. (1986). Instructions for the collection and preservation of botanical specimens. Annals of the Missouri Botanical Garden, 73(3), 504-507.

- Fonseca, R. M. (2005). Connaraceae y Resedaceae. En N. Diego-Pérez \& R. M. Fonseca (Eds.), Flora de Guerrero (23, 10 pp.). México: Universidad Nacional Autónoma de México.

- Franco-Olivares, V. H., Siqueiros-Delgado, M. E., \& HernándezAyala, E. G. (2012). Flora apícola del estado de Aguascalientes. México: Universidad Autónoma de Aguascalientes.

- Instituto Nacional de Estadística y Geografía. (2016). Anuario estadístico y geográfico de Aguascalientes 2016. Aguascalientes, México: Autor.

- Martín-Bravo, S., Vargas, P., \&, Luceño, M. (2009). Is Oligomeris (Resedaceae) indigenous to North America? Molecular evidence for a natural colonization from the Old World. American Journal of Botany, 96(2), 507-518. doi: 10.3732/ ajb.0800216

- Moreno, N. P. (1984). Glosario botánico ilustrado (llustraciones de M. Escamilla). México: Instituto Nacional de Investigaciones Sobre Recursos Bióticos.
- Nagel, P., Wurzinger, M., Iñiguez, L., Echavarría-Chairez, F. G., Flores Nájera, M. de J., Pinos-Rodríguez, J. M., ... Zollitsch, W. (2011). Sistemas de alimentacón para las cabras y evaluación cualitativa de los piensos a los que se tienen acceso durante la temporada de seca: Dos estudios de caso del Altiplano mexicano. Revista Chapingo. Serie Ciencias Forestales y del Ambiente, XVII(Esp.), 247-258. doi: 10.5154/r. rchscfa.2010.09.089

- Nee, M. (1985). Resedaceae. En A. Gómez-Pompa (Ed.), Flora de Veracruz (48, 5 pp.). México: Instituto Nacional de Investigaciones sobre Recursos Bióticos.

- Newman, M. (2007). Capparaceae Juss. En Flora Del Valle De Tehuacán-Cuicatlán (51, 11 pp.). México: Universidad Nacional Autónoma de México.

- Rzedowski, J. (2006). Vegetación de México. México: Comisión Nacional para el Conocimiento y Uso de la Biodiversidad (CONABIO). Recuperado de http://www.biodiversidad.gob.

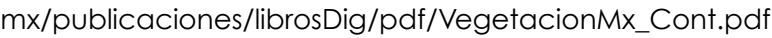

- Siqueiros-Delgado, M. E., Rodríguez-Ávalos, J. A., MartínezRamírez, J., \& Sierra-Muñoz, J. C. (2016). Situación actual de la vegetación del estado de Aguascalientes, México. Botanical Sciences, 94 (3), 455-470. doi: 10.17129/botsci.466

- Siqueiros-Delgado, M. E., Rodríguez-Ávalos, J. A., MartínezRamírez, J., Sierra-Muñoz, J. C., \& García-Regalado, G. (2017). Vegetación del estado de Aguascalientes. México: Universidad Autónoma de Aguascalientes-CONABIO.

- Standley, P. C. (1924). The genus Forchammeria. Journal of the Washington Academy of Sciences, 14, 269-272.

- Stevens, P. F. (2001). Angiosperm Phylogeny Website [Portal electrónico informativo]. Recuperado el 14 de julio de 2018 de http://www.mobot.org/MOBOT/research/APweb/

- Valdés Bermejo, E. (1993). Reseda L. En S. Castroviejo et al. (Eds.), Flora Ibérica. Plantas vasculares de la Península Ibérica e Islas Baleares: Vol. 4. Cruciferae-Monotropaceae (pp. 440473). Madrid: Real Jardín Botánico, CSIC.

- Villaseñor, J. L. (2016). Checklist of the native vascular plants of Mexico. Revista Mexicana de Biodiversidad, 87(3), 559-902. doi: 10.1016/j.rmb.2016.06.017

- Villaseñor, J. L., \& Espinosa-García, F. J. (1998). Catálogo de malezas de México. México: Universidad Nacional Autónoma de México.

(2004). The alien flowering plants of Mexico. Diversity and Distributions, 10, 113-123. doi: 10.1111/j.13669516.2004.00059.x 